\title{
Chest X-ray interpretation does not complement Xpert MTB/RIF in diagnosis of smear-negative pulmonary tuberculosis among TB-HIV co-infected adults in a resource-limited setting
}

Lydia Nakiyingi ${ }^{1,2^{*}}$ (D, John Mark Bwanika', Willy Ssengooba ${ }^{3}$, Frank Mubiru', Damalie Nakanjako², Moses L. Joloba ${ }^{3}$, Harriet Mayanja-Kizza ${ }^{2}$ and Yukari C. Manabe ${ }^{4}$

\begin{abstract}
Background: Chest X-ray (CXR) interpretation remains a central component of the current World Health Organization recommendations as an adjuvant test in diagnosis of smear-negative tuberculosis (TB). With its low specificity, high maintenance and operational costs, utility of CXR in diagnosis of smear-negative TB in high HIV/TB burden settings in the Xpert MTB/RIF era remains unpredictable. We evaluated accuracy and additive value of CXR to Xpert MTB/RIF in the diagnosis of TB among HIV-positive smear-negative presumptive TB patients.

Methods: HIV co-infected presumptive TB patients were recruited from the Infectious Diseases Institute outpatient clinic and in-patient medical wards of Mulago Hospital, Uganda. CXR films were reviewed by two independent radiologists using a standardized evaluation form. CXR interpretation with regard to TB was either positive (consistent with TB) or negative (normal or unlikely TB). Sensitivity, specificity and predictive values of CXR and CXR combined with Xpert MTB/RIF for diagnosis of smear-negative TB in HIV-positive patients were calculated using sputum and/or blood mycobacterial culture as reference standard.

Results: Three hundred sixty-six HIV co-infected smear-negative participants (female, 63.4\%; hospitalized, 68.3\%) had technically interpretable CXR. Median (IQR) age was 32 (28-39) years and CD4 count $112(23-308) \mathrm{cells} / \mathrm{mm}^{3}$. Overall, 22\% (81/366) were positive for Mycobacterium tuberculosis (Mtb) on culture; 187/366 (51.1\%) had CXR interpreted as consistent with TB, of which 55 (29.4\%) had culture-confirmed TB. Sensitivity and specificity of CXR interpretation in diagnosis of culture-positive TB were $67.9 \%$ (95\%Cl 56.6-77.8) and 53.7\% (95\%Cl 47.7-59.6) respectively, while Xpert MTB/RIF sensitivity and specificity were $65.4 \%(95 \% \mathrm{Cl} 54.0-75.7)$ and $95.8 \%(95 \% \mathrm{Cl} 92.8-$ 97.8) respectively. Addition of CXR to Xpert MTB/RIF had overall sensitivity and specificity of $87.7 \%(95 \% \mathrm{Cl} 78.5-93.9)$ (Continued on next page)
\end{abstract}

\footnotetext{
* Correspondence: Lydiakiyingi@gmail.com

${ }^{1}$ Research Department, Infectious Diseases Institute, Makerere University College of Health Sciences, Kampala, Uganda

${ }^{2}$ Department of Medicine, School of Medicine, Makerere University College of Health Sciences, Kampala, Uganda

Full list of author information is available at the end of the article
}

(c) The Author(s). 2021 Open Access This article is licensed under a Creative Commons Attribution 4.0 International License, which permits use, sharing, adaptation, distribution and reproduction in any medium or format, as long as you give appropriate credit to the original author(s) and the source, provide a link to the Creative Commons licence, and indicate if changes were made. The images or other third party material in this article are included in the article's Creative Commons licence, unless indicated otherwise in a credit line to the material. If material is not included in the article's Creative Commons licence and your intended use is not permitted by statutory regulation or exceeds the permitted use, you will need to obtain permission directly from the copyright holder. To view a copy of this licence, visit http://creativecommons.org/licenses/by/4.0/. The Creative Commons Public Domain Dedication waiver (http://creativecommons.org/publicdomain/zero/1.0/) applies to the data made available in this article, unless otherwise stated in a credit line to the data. 


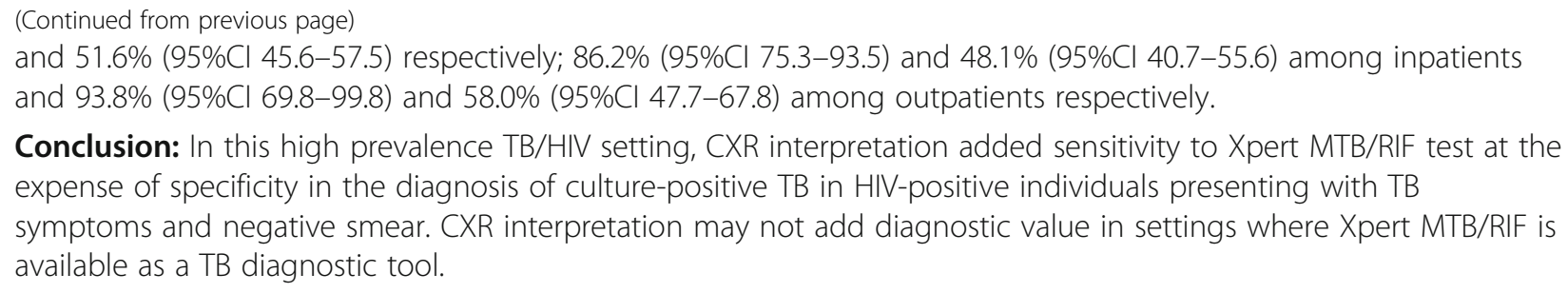

Conclusion: In this high prevalence TB/HIV setting, CXR interpretation added sensitivity to Xpert MTB/RIF test at the expense of specificity in the diagnosis of culture-positive TB in HIV-positive individuals presenting with TB symptoms and negative smear. CXR interpretation may not add diagnostic value in settings where Xpert MTB/RIF is available as a TB diagnostic tool.

Keywords: Chest X-ray, Resource-limited setting, Tuberculosis, HIV, Diagnosis, Smear-negatives, Uganda

\section{Background}

Tuberculosis (TB) remains a significant public health problem globally and is the main cause of death among persons living with HIV [1]. Key global priorities for TB care and control emphasize improving earlier case detection and linkage to care. Globally, in 2018, a total of 10 million cases of TB were estimated, of which only 6.4 million (64\%) were diagnosed and notified to national programs, the remaining $36 \%$ of the estimated TB cases were left unreported [1]. These unreported and hence untreated cases are a reservoir of infection with ongoing transmission and contribute significantly to the persistently high TB prevalence and mortality particularly among HIV co-infected individuals [1-3]. A recent study showed that in resource-limited settings (RLS), TB accounted for approximately $40 \%$ of facility-based HIV/ AIDS-related adult deaths, and that in almost half of these, TB was undiagnosed at the time of death [4].

In 2018, Uganda had an estimated TB incidence of 86, 000 cases with $24 \%$ deaths. In the same year, 34,000 of the new cases were HIV and TB co-infected with an estimated 11,000 HIV-infected individuals having died from $\mathrm{TB}$, making Uganda one of the most affected countries worldwide for TB/HIV co-infection. Of the estimated new TB cases in Uganda, only $65 \%$ were notified to the national TB program in 2018 [1, 5]. Delayed and missed TB diagnosis in HIV-positive individuals is largely responsible for the high mortality reported in many RLS endemic for TB/HIV in sub-Saharan Africa (SSA) [6-9].

In many RLS, sputum smear microscopy is still the most widely used up-front diagnostic method at most low-level health centers, and yet it only detects about half of TB cases especially in immunocompromised patients [10]. Low accuracy of sputum smear microscopy has resulted into high frequency of smearnegative $\mathrm{TB}$ and increased reliance on empiric decision-making to initiate TB treatment in HIV coinfected patients $[11,12]$.

In order to achieve the World Health Organization (WHO) goal of ending the TB epidemic by 2035 [13], we need to identify missing TB cases, link them and retain them in appropriate care. One of the strategies to improve identification of these missing cases is improved diagnosis including application of new TB diagnostic tools that have been found to have improved performance. The Xpert MTB/RIF and Ultra assays on the GeneXpert platform (Cepheid, Sunnyvale, CA) have shown the potential to provide rapid, sensitive diagnosis and have been rolled out in several countries in SSA $[14,15]$. However, implementation of Xpert MTB/RIF is costly for most low-income countries and requires sophisticated infrastructure that may not be readily accessible in most resource-limited settings [16-18]. Additionally, although the sensitivity of Xpert MTB/RIF assay for pulmonary $\mathrm{TB}$ detection was found to be approximately $98 \%$ for sputum smear-positive individuals, the sensitivity is much lower at $67-75 \%$ for sputum smear-negative HIV-positive individuals $[19,20]$. This highlights the need for adjuvant tests and empiric decision-making for smear-negative HIV-positive individuals in particular.

The WHO still considers CXR an important piece in TB diagnosis particularly as an adjuvant test in smearnegative TB diagnostic algorithms [21, 22]. National TB guidelines for most RLS have included use of CXR as part of diagnostic algorithms to complement clinical diagnosis for $\mathrm{TB}$ especially for patients with negative sputum smear and/or Xpert MTB/RIF [5, 22]. However, implementation of CXR needs resources to meet operational costs related to electricity, X-ray films, CXR maintenance, and good real-estate facilities that are frequently not available in most RLS. Paucity of personnel that can correctly interpret CXR at most health facilities further challenges CXR implementation [23]. Moreover, the low specificity of CXR is often associated with overdiagnosis of TB, which results in unnecessary TB treatment, wastage of resource and unnecessary toxicity [2326]. In this Xpert MTB/RIF era, National TB programs in low-income countries need to make important policy decisions on allocation of resources and positioning of CXR within the TB diagnostic algorithms. There is limited published data available on the role of CXR in the Xpert MTB/RIF era to guide policymakers in RLS.

We evaluated the sensitivity, specificity, predictive values and likelihood ratios of CXR interpretation as stand-alone and when in combination with Xpert MTB/ RIF, in the diagnosis of smear-negative TB among HIV 
co-infected patients presenting with symptoms of TB. The goal was to determine the additive value of CXR to smear or Xpert MTB/RIF test in the diagnosis of smearnegative TB among HIV-positive individuals in a high HIV/TB burden setting.

\section{Methods}

\section{Study design and setting}

This study was among participants that were enrolled in a TB diagnostics study that evaluated the lateral flow TB-lipoarabinomannan (TB-LAM) test for the diagnosis of TB among HIV-infected patients with signs and symptoms of TB [27]. The TB diagnostics study recruited outpatients at the adult Infectious Diseases Institute (IDI) Clinic and inpatients at the medical wards of Mulago National Referral Hospital, Kampala Uganda, between January and November 2011.

\section{Patient recruitment and study population}

As previously described [27, 28], the study involved HIV-infected patients at the age of 18 years and above, who were clinically suspected to have active TB (presumptive TB) based on presence of at least one of cough, fever, drenching night sweats or weight loss within the past 4 weeks of enrolment, as reported by the patient. Patients who had taken anti-TB medication for more than 2 days within 60 days prior to enrolment were excluded.

Our previous publications describe the details of the study procedures $[27,28]$. In summary, enrolled participants were interviewed for socio-demographic and medical data before study samples were collected. Eligible participants provided two spot sputum samples for conventional $\mathrm{TB}$ tests that included; direct fluorescence microscopy (FM) and Ziehl-Neelson (ZN) microscopy; mycobacterial growth indicator tube (MGIT) and Lowenstein-Jensen (LJ) sputum cultures. For participants who were unable to spontaneously expectorate sputum, trained study personnel performed sputum induction. Sputum collection and induction were performed in a separately designated and well-aerated designated area without other patients or staff in the immediate area. The study staff used protective facemasks, gloves and disposable aprons during the entire course of the procedure. Mycobacterial blood cultures and CD4 cell count were performed on blood specimens that were collected at enrolment. Urine TB-LAM testing using a lateral flow assay (TB-LAM Alere, Waltham, MA, USA) was also performed. All male participants and women with a confirmed negative urine pregnancy test had a chest X-ray (CXR) performed.

Sputum smear-negative participants were those that were smear-negative on both $\mathrm{ZN}$ and FM microscopy [21], while MTB culture-positives were those that were
MTB positive on any of sputum and/or blood mycobacterial cultures. For this study (Fig. 1), only sputum smear-negative HIV-infected patients with technically adequate CXR were included. The remaining inclusion and exclusion criteria were consistent with the existing study as described above. Participants without MTB culture results for reference were also excluded from this analysis. Sputum and blood TB cultures were used as reference standard.

\section{Clinical data}

The following general characteristics were collected at enrollment: age, gender, CD4 count $\left(\right.$ cells $\left./ \mathrm{m}^{3}\right)$, sputum smear microscopy, hospital setting, Karnofsky score. In order to assess the participant medical status at the time of visit, antiretroviral (ARV) treatment and cotrimoxazole use, previous TB treatment and previous ARV were obtained. Clinical symptoms that included cough, fever, weight loss, anorexia, excessive night sweats, difficulty in breathing and lymphadenopathy were collected. Cough, fever, weight loss, anorexia and excessive night sweats were retrieved as subjective symptoms that occurred within the past 4 weeks as reported by the patient. All characteristics were ascertained at enrolment.

\section{CXR radiographs interpretation procedure}

Chest X-Rays (CXRs) were performed at enrolment for all study participants except pregnant females. CXR were reviewed by two independent experienced radiologists using a standardized CXR evaluation form. If the two reports differed, a third radiologist was used as a tiebreaker. All readers were blinded to the laboratory data, clinical findings and final diagnosis of the patient. The radiographic features analyzed were infiltrates (upper/mid/lower), fibrosis, cavity, miliary disease, adenopathy, pleural effusion, pleural thickening and others. Based on the general impression of the radiologist, CXR interpretation with regard to TB was reported as either positive (consistent with $\mathrm{TB}$ ) or negative (normal or unlikely $\mathrm{TB}$ ) as the main outcome.

\section{Laboratory procedures}

To identify sputum smear-negative participants, smear microscopy was performed on two spot sputum samples, using FM and ZN. Participants were classified as sputum smear-negative if they were smear-negative on both $\mathrm{ZN}$ and FM microscopy. The same sputum samples were used to perform MGIT and LJ cultures. Each participant in addition had a mycobacterial blood culture. A positive MTB culture was defined as presence of at least one positive $\mathrm{TB}$ culture on any of the three $\mathrm{TB}$ cultures MGIT, LJ or MTB blood culture. Details of the 


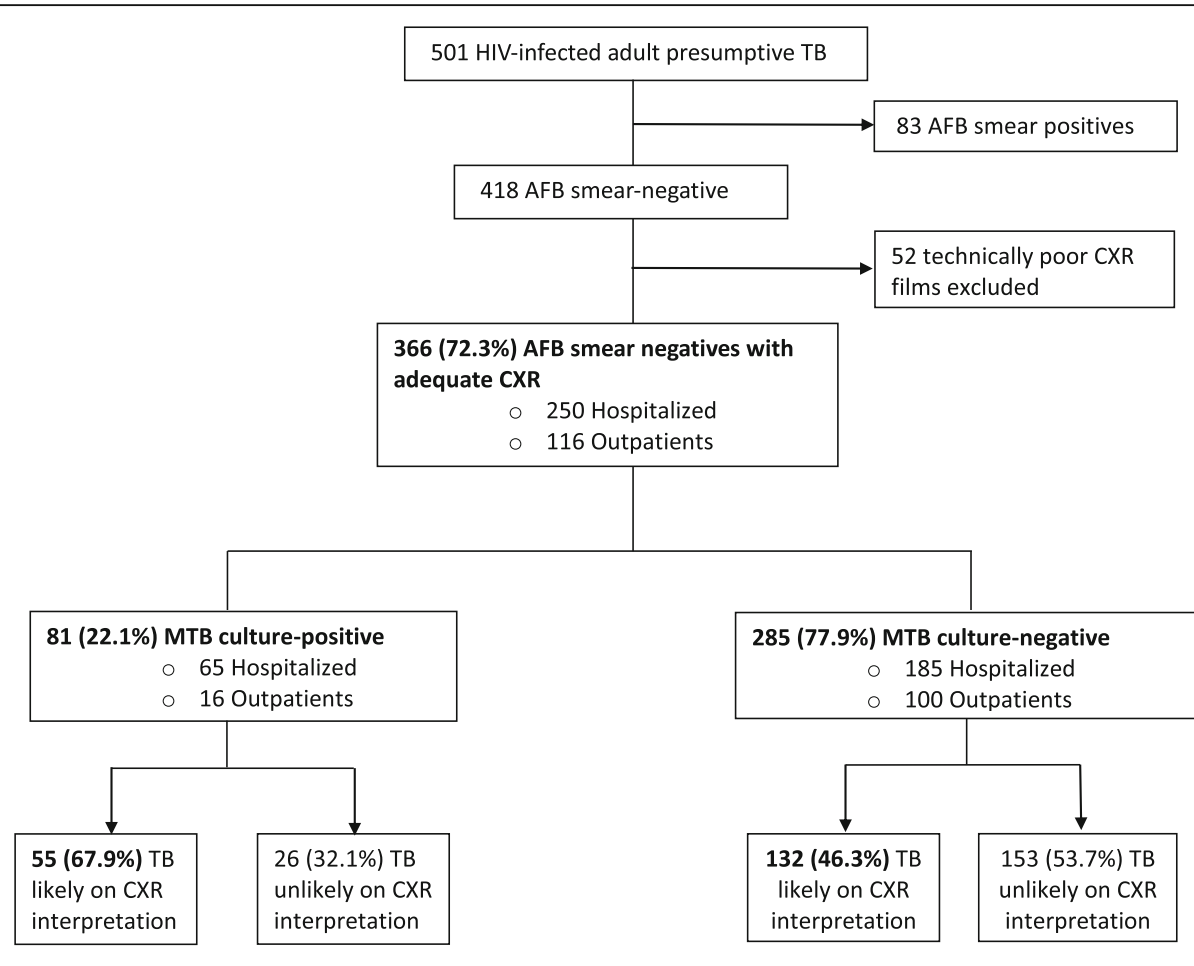

Abbreviations: AFB, Acid-Fast Bacilli; TB, tuberculosis; CXR, chest X-ray; MTB, Mycobacterium tuberculosis.

Fig. 1 Participant flow chart

laboratory procedures for all study tests have been described earlier $[27,28]$. All confirmed MTB positive patients by any of the TB tests performed during the study were promptly initiated on TB treatment by the attending clinician according to the Uganda Ministry of Health $\mathrm{TB}$ and Leprosy program guidelines [5].

\section{Statistical analysis}

The analysis was performed on data from a subset of participants enrolled in a TB diagnostic accuracy study of the lateral flow TB-lipoarabinomannan (TBLAM) test for the diagnosis of $\mathrm{TB}$ among HIVinfected presumptive TB patients. All statistical analyses were performed using Stata version 12.0. Categorical data were summarised using frequencies, proportions and percentages. Continuous data were summarised using means and median. Accuracy (sensitivity, specificity, positive and negative predictive values as well as likelihood ratio positive and negative) of CXR interpretation and when combined with Xpert MTB/RIF in the diagnosis of smear-negative TB was calculated using sputum and/or blood TB cultures as reference standard. All statistics were twotailed and $p$-values less than 0.05 were considered statistically significant.

\section{Results}

\section{Baseline characteristics}

Of the $501 \mathrm{HIV}$-infected presumptive TB patients who provided sputum samples for microscopy, 418 (83.4\%) were sputum smear-negative. Of the 418 smearnegatives, 366 with MTB culture results available had technically adequate chest radiographs and thus were included in this analysis (Fig. 1). Of the 366 sputum smear-negative participants eligible for analysis, $68.3 \%$ (250/366) were inpatients, $63.4 \%(232 / 366)$ were female; median age was 32 (IQR 28, 39) years and median CD4 count was 112 (IQR 23-308) cells $/ \mathrm{mm}^{3}$.

\section{MTB culture results}

Of the 366 sputum smear-negative participants analyzed, 81/366 (22.1\%) were positive for Mycobacterium tuberculosis (MTB) complex on blood and/or sputum TB cultures; of which, 48/81 (59.3\%) were MTB culture positive on sputum alone, $28 / 81$ (34.6\%) were positive on both sputum and blood cultures while 5/81 (6.2\%) were positive on blood culture alone. Majority of the MTB culture-positives were inpatients (65/81, 80.2\%). The median CD4 cell count was significantly lower in the MTB culture-positive compared to the culturenegative participants [79 (IQR 15-179) versus 130 (IQR 
26-331) cells $/ \mathrm{mm}^{3}, p=0.011$ respectively]. A comparison of clinical and laboratory characteristics between MTB culture-positive and negative participants is shown in Table 1.

\section{Accuracy of chest X-ray interpretation}

Overall, of the 366 participants with interpretable CXR, 187/366 (51.1\%) had CXR interpreted as consistent with pulmonary TB, of which only 55/187 (29.4\%) had MTB culture confirmed TB on sputum and/or blood (Table 1). Abnormal CXR findings interpreted as TB were more frequent among the MTB culture positive compared to MTB culture-negative smear-negative HIV participants (67.9\% versus $46.3 \% p=0.001)$. The most frequent abnormalities reported include; lower lobe infiltrates (41\%), upper and middle lobe infiltrates (39.1\%), and pleural disease including pleural effusion, $10.7 \%$ and pleural thickening, 9.6\%. All these CXR abnormalities were more common among MTB culture positives (Table 1).
Miliary TB (1.6\%) and cavities (4.1\%) were rare among the participants and majority (4 out of 6 ) of the miliary TB participants on CXR also had positive MTB cultures but negative urine TB-LAM.

The sensitivity and specificity of CXR interpretation determined using MTB culture as reference standard in the diagnosis of smear-negative TB in HIV-co infected individuals were $67.9 \%$ (95\% CI 56.6-77.8\%) and 53.7\% (95\% CI 47.7-59.6\%) respectively (Table 2 ). The positive and negative predictive values were $29.4 \%$ (95\% CI $23-$ $36.5 \%)$ and $85.5 \%$ (95\% CI 79.4-90.3\%) respectively.

Restricting the analysis to inpatients, $135 / 250$ (54\%) had CXR interpreted as consistent with TB, of which 43/ 135 (31.9\%) were MTB culture positive. The sensitivity and specificity of CXR interpretation among inpatient participants were therefore $66.2 \%$ (95\%CI 53.4-77.4\%) and $50.3 \%$ (95\% CI 42.8-57.7\%) respectively. Analysis among the outpatients alone found, of the 116 outpatients, 52 (54\%) had CXR interpreted as consistent with

Table 1 Characteristics of HIV-positive, smear-negative presumptive participants, comparing MTB culture-positives and negatives $(n=366)$

\begin{tabular}{|c|c|c|c|c|}
\hline Participants characteristics & Overall $(N=366)$ & Culture positives $(N=81)$ & Culture negatives $(N=285)$ & $P$-value \\
\hline \multicolumn{5}{|l|}{ Clinical characteristics } \\
\hline Age at visit time (Median (IQR) & $32(28,39)$ & $31(27,37)$ & $33(28,40)$ & 0.064 \\
\hline CD4 count (cells/mm3) (Median, IQR) & $112(23,308)$ & $79(15,179)$ & $130(26,331)$ & 0.011 \\
\hline Gender: Female $(n, \%)$ & $232(63.4)$ & $43(53.1)$ & $189(66.3)$ & 0.029 \\
\hline Inpatient participants & $250(68.3)$ & $65(80.3)$ & $185(64.9)$ & 0.009 \\
\hline Cough Present & $359(98.1)$ & $80(98.8)$ & $279(97.9)$ & 1.000 \\
\hline Fever present & $333(91.0)$ & 75 (92.6) & $258(90.5)$ & 0.665 \\
\hline Weight loss present & $325(88.8)$ & $80(98.8)$ & $245(86.0)$ & 0.001 \\
\hline \multicolumn{5}{|l|}{ Medication history } \\
\hline Antiretroviral therapy $(n, \%)$ & $137(37.4)$ & $24(29.6)$ & 113 (39.6) & 0.037 \\
\hline Cotrimoxazole Prophylaxis & $341(93.2)$ & $78(96.3)$ & $263(92.3)$ & 0.299 \\
\hline Previous medication for TB disease & $67(18.3)$ & $9(11.1)$ & $58(20.4)$ & 0.032 \\
\hline \multicolumn{5}{|l|}{ TB tests results } \\
\hline Xpert MTB/RIF positive & $65(17.8)$ & $53(65.4)$ & $12(4.2)$ & 0.000 \\
\hline Urine TB LAM Positive (n, \%) & $50(13.7)$ & $28(34.6)$ & $22(7.7)$ & 0.000 \\
\hline CXR interpreted as TB (n, \%) & $187(51.1)$ & $55(67.9)$ & $132(46.3)$ & 0.001 \\
\hline \multicolumn{5}{|l|}{ Radiological Characteristics (n, \%) } \\
\hline Upper infiltrates $^{a}$ & $143(39.1)$ & $52(64.2)$ & $91(31.9)$ & 0.000 \\
\hline Lower Infiltrates & $150(41.0)$ & $46(56.8)$ & $104(36.5)$ & 0.001 \\
\hline Cavity & $15(4.1)$ & $2(2.5)$ & $13(4.6)$ & 0.537 \\
\hline Miliary disease & $6(1.6)$ & $4(4.9)$ & $2(0.7)$ & 0.023 \\
\hline Pleural Effusion & $39(10.7)$ & $13(16.0)$ & $26(9.1)$ & 0.100 \\
\hline Pleural Thickening & $35(9.6)$ & $12(14.8)$ & $23(8.1)$ & 0.085 \\
\hline Adenopathy & $13(3.6)$ & $5(6.2)$ & $8(2.8)$ & 0.172 \\
\hline Fibrosis & $20(5.5)$ & $4(4.9)$ & $16(5.6)$ & 1.000 \\
\hline
\end{tabular}

Abbreviations: TB Tuberculosis; ART Antiretroviral therapy; CXR Chest X-ray; LAM Lipoarabinomannan; IQR Inter-quartile range; ${ }^{a}$ means infiltrates in upper or middle lobe 
Table 2 Accuracy of CXR interpretation and when combined with Xpert MTB/RIF in HIV-related smear-negative TB diagnosis

\begin{tabular}{|c|c|c|c|c|}
\hline Setting & Accuracy Index & CXR Interpretation & Xpert MTB/RIF test & CXR plus Xpert MTB/RIF test \\
\hline \multirow[t]{7}{*}{ Overall } & Sensitivity (95\% CI) & $67.9 \%(56.6-77.8)$ & $65.4 \%(54.0-75.7)$ & $87.7 \%(78.5-93.9)$ \\
\hline & Specificity $(95 \% \mathrm{Cl})$ & $53.7 \%(47.7-59.6)$ & $95.8 \%(92.8-97.8)$ & $51.6 \%(45.6-57.5)$ \\
\hline & PPV $(95 \% \mathrm{Cl})$ & $29.4 \%(23.0-36.5)$ & $81.5 \%(70.0-90.1)$ & $34.0 \%(27.6-40.8)$ \\
\hline & NPV $(95 \%$ Cl) & $85.5 \%(79.4-90.3)$ & $90.7 \%(86.8-93.7)$ & $93.6 \%(88.6-96.9)$ \\
\hline & Likelihood ratio (+) & $1.47(1.21-1.78)$ & $15.54(8.74-27.64)$ & $1.81(1.57-2.09)$ \\
\hline & Likelihood ratio $(-)$ & $0.60(0.43-0.84)$ & $0.36(0.27-0.49)$ & $0.24(0.13-0.43)$ \\
\hline & Area under ROC curve & $0.61(0.55-0.67)$ & $0.81(0.75-0.86)$ & $0.70(0.65-0.74)$ \\
\hline \multirow[t]{7}{*}{ Inpatients } & Sensitivity & $66.2 \%(53.4-77.4)$ & $64.6 \%(51.8-76.1)$ & $86.2 \%(75.3-93.5)$ \\
\hline & Specificity & $50.3 \%(42.8-57.7)$ & $95.7 \%(91.7-98.1)$ & $48.1 \%(40.7-55.6)$ \\
\hline & PPV (95\% Cl) & $31.9 \%(24.1-40.4)$ & $84.0 \%(70.9-92.8)$ & $36.8 \%(29.2-45.0)$ \\
\hline & NPV $(95 \% \mathrm{Cl})$ & $80.9 \%(72.5-87.6)$ & $88.5 \%(83.2-92.6)$ & $90.8 \%(83.3-95.7)$ \\
\hline & Likelihood ratio (+) & $1.33(1.06-1.67)$ & $14.94(7.41-30.13)$ & $1.66(1.40-1.97)$ \\
\hline & Likelihood ratio (-) & $0.67(0.47-0.97)$ & $0.37(0.27-0.51)$ & $0.29(0.15-0.54)$ \\
\hline & Area under ROC curve & $0.58(0.51-0.65)$ & $0.80(0.74-0.86)$ & $0.67(0.62-0.73)$ \\
\hline \multirow[t]{7}{*}{ Outpatient } & Sensitivity & $75.0 \%(47.6-92.7)$ & $68.8 \%(41.3-89.0)$ & $93.8 \%(69.8-99.8)$ \\
\hline & Specificity & $60.0 \%(49.7-69.7)$ & $96.0 \%(90.1-98.9)$ & $58.0 \%(47.7-67.8)$ \\
\hline & PPV (95\% Cl) & $23.1 \%(12.5-36.8)$ & $73.3 \%$ (44.9-92.2) & $26.3 \%(15.5-39.7)$ \\
\hline & NPV (95\% Cl) & $93.8 \%(84.8-98.3)$ & $95.0 \%(88.8-98.4)$ & $98.3 \%(90.9-100)$ \\
\hline & Likelihood ratio (+) & $1.88(1.29-2.72$ & $17.19(6.23-47.45)$ & $2.23(1.72-2.90)$ \\
\hline & Likelihood ratio (-) & $0.42(0.18-0.99)$ & $0.33(0.16-0.67)$ & $0.11(0.02-0.72)$ \\
\hline & Area under ROC curve & $0.67(0.56-0.79)$ & $0.82(0.70-0.94)$ & $0.76(0.68-0.84)$ \\
\hline
\end{tabular}

Abbreviations: Cl Confidence Intervals; CXR Chest X-ray; PPV Positive predictive value; NPV Negative predictive value; (+),Positive; (-),Negative; ROC Receiver Operating Characteristic curve

Table 2 shows accuracy of CXR interpretation and when combined with Xpert MTB/RIF for the diagnosis of smear-negative TB in HIV using Mycobacterial cultures as reference standard.

TB, of which 12/52 (23.1\%) were MTB culture positive. The sensitivity and specificity of CXR interpretation among the outpatient participants were $75 \%$ (95\% CI 47.6-92.7\%) and 60\% (95\% CI 49.7-69.7\%) respectively (Table 2).

\section{Accuracy of Xpert MTB/RIF test}

Xpert MTB/RIF test was positive in 65 (17.8\%) of the study participants; $53(65.4 \%)$ were among the 81 MTB culture positives and $12(4.2 \%)$ among the 285 MTB culture negatives. Therefore, for the diagnosis of smear-negative TB among HIV co-infected participants, Xpert MTB/RIF test had overall sensitivity and specificity of $65.4 \%$ (95\%CI $54.0-75.7 \%$ ) and $95.8 \%$ (95\%CI 92.8-97.8\%) respectively (Table 2). Among inpatients only, Xpert MTB/RIF sensitivity and specificity were $64.6 \% \quad$ (95\%CI $51.8-76.1 \%)$ and $95.7 \%$ (95\%CI 91.7-98.1\%) respectively while among outpatients, the sensitivity and specificity were $68.8 \%$ (95\%CI 41.3-89\%) and 96\% (95\%CI 90.1-98.9\%) respectively (Table 2).

\section{Incremental accuracy of CXR to Xpert MTB/RIF}

Addition of CXR interpretation to Xpert MTB/RIF test diagnosed 209 (57.1\%) of the $366 \mathrm{HIV}$-infected smearnegatives as pulmonary $\mathrm{TB}$, of which 71/209 (34\%) participants had MTB culture confirmed TB. Using MTB culture as reference standard, the sensitivity of Xpert MTB/RIF when CXR interpretation was added for the diagnosis of TB among smear-negative HIV-infected patients was $87.7 \%(95 \% \mathrm{CI} 78.5-93.9 \%)$ and the specificity was $51.6 \%$ (95\%CI $45.6-57.5 \%)$. The positive and negative predictive values were $34 \%$ (95\% CI 27.6-40.8\%) and 93.6\% (95\% CI 88.6-96.9\%) respectively.

Among inpatients, the sensitivity and specificity of Xpert MTB/RIF when CXR interpretation was added for the diagnosis of culture confirmed TB among HIVinfected smear-negatives were $86.2 \%$ (95\%CI $75.3-$ 93.5\%) and 48.1\% (95\%CI 40.7-55.6\%) respectively, while among outpatients, the sensitivity and specificity were 93.8\% (95\%CI 69.8-99.8\%) and 58\% (95\% CI 47.7$67.8 \%$ ) respectively (Table 2 ).

Further analysis involving only TB treatment naive patients did not show any improvement in the 
performance of CXR in the diagnosis of PTB among TB treatment naïve smear-negative HIV patients (sensitivity 67.6, 95\% CI 55.7-78.0\%; specificity 59.6, 95\% CI 52.8$66.0 \%)$. Additionally, compared to the overall participants, no significant difference in incremental yield was observed when CXR was used in combination with Xpert MTB/Rif in the population of TB treatment naïve patients (sensitivity 86.5 95\%CI $76.5-93.3 \%$ and specificity 57.3 95\% CI 50.6-63.9\%) (Table 3).

\section{Discussion}

Chest X-Ray (CXR) remains a useful adjuvant test in smear-negative pulmonary $\mathrm{TB}$ to guide further patient management in high TB/HIV prevalence settings. Given the recent widespread roll-out of Xpert MTB/RIF in most low-income countries, we evaluated the role of CXR in the diagnosis of smear-negative TB among HIVpositive presumptive TB patients in the Xpert MTB/RIF era. Overall, we found poor performance of CXR interpretation in the diagnosis of culture- positive smearnegative TB among HIV-positive participants. We found that, although CXR increased sensitivity of Xpert MTB/ RIF assay, this was at the expense of specificity. Given its high negative predictive value, a normal CXR could reliably exclude TB in HIV-positives with negative sputum smear, however, the applicability of CXR in settings with Xpert MTB/Rif is limited by the challenges of CXR application faced in most RLS. Our study further found that combining CXR with Xpert MTB/RIF reduced the specificity of Xpert MTB/RIF in the diagnosis of smearnegative TB. This implies that use of CXR as an adjuvant to Xpert MTB/RIF results into inaccurate diagnosis of TB among HIV co-infected presumptive TB patients with negative smear.

Our findings corroborate with earlier reports from high prevalence TB/HIV settings in SSA which also found poor performance, particularly low specificity, of CXR interpretation in the diagnosis of cultureconfirmed TB among smear-negative HIV co-infected individuals [24, 26, 29-31].
Poor performance of CXR in TB diagnosis in HIV coinfected individuals is often attributed to the nonspecific CXR patterns and difficulty in interpretation [23, 25]. Results of a chest radiograph often depend on the severity and presentation of TB, which all depend on the stage of the HIV disease, with advanced HIV disease more likely to present with non-specific patterns compared to early-stage HIV disease. Moreover, CXR findings can be normal in up to $25-50 \%$ of patients with culture-confirmed TB $[23,25,26]$. Our study population comprised HIV co-infected individuals, majority of whom had advanced HIV disease (median CD4 count 112 cells $/ \mathrm{mm}^{3}$ ) and this could potentially explain the non-specific patterns inaccurately interpreted as $\mathrm{TB}$, and thus the low specificity seen in our study.

CXR interpretation in HIV co-infection is further complicated by the presence of other HIV-related pulmonary diseases that could radiologically mimic TB; commonly, bacterial pneumonia [23, 24]. Several studies from SSA $[24,26,30]$ have reported presence of other HIV-related pulmonary conditions that radiologically mimic TB that have led to misinterpretation of chest radiographs as TB. This too could explain the poor CXR performance in this study that was performed among entirely HIV-positive individuals. However, our study did not evaluate other possible non-TB pulmonary conditions that could explain the inaccurate CXR interpretation.

Poor performance of CXR interpretation in this study could also be explained by poor quality of CXR films, which in turn is dependent on the skill of the technician, and the quality of the CXR equipment used. Studies have shown accuracy of CXR interpretation is dependent on the quality of the CXR film [23, 30]. In most peripheral health centres in RLS where CXR services are available, the quality of the radiographs produced from the poor quality CXR equipment is often not good enough to improve interpretation [23]. To improve accuracy of CXR interpretation in our study, CXR interpretation was performed by expert radiologists.

Table 3 Accuracy of CXR and CXR plus GeneXpert in smear-negative TB diagnosis among TB-treatment naïve participants

\begin{tabular}{lll}
\hline Accuracy index & CXR Interpretation & CXR plus Xpert MTB/RIF test \\
\hline Sensitivity (95\% Cl) & $67.6 \%(55.7-78)$ & $86.5 \%(76.5-93.3)$ \\
Specificity (95\% Cl) & $59.6 \%(52.8-66)$ & $57.3 \%(50.6-63.9)$ \\
PPV (95\% Cl) & $35.5 \%(27.6-44)$ & $40 \%(32.3-48)$ \\
NPV (95\% Cl) & $84.8 \%(78.2-90)$ & $92.8 \%(87.2-96.5)$ \\
Likelihood ratio (+) & $1.67(1.34-2.09)$ & $2.03(1.7-2.42)$ \\
Likelihood ratio (-) & $0.545(0.385-0.77)$ & $0.236(0.131-0.424)$ \\
AUC (ROC area) & $0.636(0.573-0.698)$ & $0.719(0.668-0.77)$ \\
\hline
\end{tabular}

CI Confidence Intervals; CXR Chest X-ray; PPV Positive predictive value; NPV Negative predictive value; (+),Positive; (-), Negative; ROC Receiver Operating Characteristic curve

Table 3 shows accuracy of CXR interpretation and when CXR is combined with Xpert MTB/RIF for the diagnosis of smear-negative TB among TB treatment naive HIV-positive patients using Mycobacterial cultures as reference standard. 
Our study further found that CXR interpretation did not complement Xpert MTB/RIF diagnostic performance in smear-negative TB diagnosis in this low-income country with high TB/HIV prevalence. Instead, we noted decreased accuracy with loss in specificity of Xpert MTB/RIF test when additively used with CXR interpretation i.e. Xpert MTB/RIF specificity dropped from $95.8 \%$ when used as stand-alone test to $51.6 \%$ when additively used with CXR interpretation. A similar trend was seen when results were stratified by setting (outpatients versus inpatients). Our findings are similar to an earlier report, which also found a reduction in specificity of Xpert MTB/RIF when additively used with CXR interpretation [31]. The reduction in specificity is attributable to the non-specific nature of CXR patterns in HIV disease, which results into inaccurate interpretation $[23,26]$. These findings imply that use of CXR interpretation as an additive test to Xpert MTB/RIF test in centers where Xpert MTB/RIF is available as a TB diagnostic increases the likelihood of inaccurate diagnosis of TB, which consequently puts more financial strain to TB programs in RLS and leads to misuse of already scarce resources. However, based on our recent findings from the same patient population, CXR could complement urine TB Lipoarabinomannan (LAM) if Xpert MTB/RIF is not available [28]. The previous findings showed that an algorithm in which CXR is performed following urine TB LAM test significantly improves diagnosis of TB among smear-negative HIV-positive patients with a high negative predictive value, especially when CD4 counts are < 100cells $/ \mathrm{mm}^{3}$ [28]. This was not the case when CXR was combined with Xpert MTB/RIF test in the diagnosis of TB in smear-negative HIV-positive patients.

Of note, our study found lower lung field infiltrates (41\%) and pleural disease among the most frequent abnormalities reported by the expert radiologist. This is similar to earlier findings from other studies on CXR features among HIV co-infected individuals [23-26, 30]. These atypical CXR features are not surprising in our study, which included entirely HIV co-infected patients, many of whom had immunologically advanced HIV disease. Advanced HIV disease could also explain the miliary TB disease CXR pattern seen in a few of our study patients. Cavities were rare among our study participants as is expected in HIV-positive patients with advanced disease $[23,25]$.

This study had strengths. First, our study included both sputum and blood MTB culture as the standard of reference, which improved the strength of our reference standard for TB diagnosis. Inadequacy of gold standard is often a limitation in TB diagnostics evaluations. Second, in order to improve CXR interpretation, we used two expert radiologists to interpret the chest radiographs, and in case of variability, a third radiologist was used as a tiebreaker. Third, our study population and setting well represent a real-world situation that is likely to be found in high prevalence HIV/TB settings in lowincome countries. We studied a population of HIVinfected individuals with an increased risk of morbidity and mortality from $\mathrm{TB}$ that is targeted in the End $\mathrm{TB}$ strategy. Lastly, we used a relatively simple CXR evaluation form in the interpretation of the CXR, which is a more practical approach since hospitals in RLS often lack personnel that can interpret CXR with expertise and are often understaffed. By using a simple and easyto-use CXR interpretation form, we assigned the accuracy of the CXR in this study more agreeable with resource-constrained settings. However, the study results cannot be generalized to populations that were not included in this study, particularly pregnant women and children.

\section{Conclusion and recommendation}

In this high HIV/TB burden resource-limited setting, CXR interpretation had low diagnostic utility in HIV coinfected patients presenting with $\mathrm{TB}$ symptoms and negative smear. Addition of CXR to Xpert MTB/RIF increased sensitivity but reduced specificity in the diagnosis of smear-negative TB among HIV-positive patients. CXR interpretation may not add diagnostic value in settings where Xpert MTB/RIF is available as a TB diagnostic tool.

\section{Abbreviations}

Cl: Confidence interval; CXR: Chest x-ray; FM: Fluorescence microscopy; IRB: Institutional review board; LJ: Lowenstein-jensen; MGIT: Mycobacterial growth indicator tube; MTB: Mycobacterium tuberculosis; TB: Tuberculosis; TBLAM: TB-lipoarabinomannan; ZN: Ziehl-neelson

\section{Acknowledgements}

The authors would like to thank the TB diagnostics research team at the Infectious Diseases Institute (IDI) of the College of Health Sciences, Makerere University, particularly Esther Namanda, Elizabeth Nakabugo, Jessica Nanyombi, Betty Namboozo, Esther Eliamo, Bernadette Nakawooya and the Mycobacteriology (BSL-3) Laboratory of Makerere University for their important contributions to the implementation of this study. The authors would also wish to thank the radiologists led by Dr. Harriet Kisembo who participated in the CXR interpretation. The authors gratefully acknowledge the study participants for their willingness to participate in the study.

\section{Authors' contributions}

$L N, Y C M, M L$, HMK conceived the idea; $L N, Y C M, D N$ obtained funding, $L N$, $J M B$, WS, acquired the data; LN, FM, MJ, JMB, HMK, DN, YCM performed the analysis and interpretation, LN primarily drafted the article. All authors contributed to revising this manuscript and gave final approval of the version.

\section{Funding}

LN is a post-doctoral fellow supported by Grant Number D43TW010132 supported by office of the Director, National Institute of Health, National Institute of Dental \& Craniofacial Research (NIDCR), National Institute of Neurological Disorders and Stroke (NINDS), National Heart, Lung and Blood Institute (NHLBI), Fogarty International Center (FIC), National Institute on Minority Health and Health Disparities (NIMHD). Additional support to LN's postdoctoral fellowship program was through D43TW009771 and D43TW010132 from the Fogarty International Center, National Institutes of Health to YCM. 
Additional funding to DN through MUII-plus from Wellcome Trust grant number 107743/z/15/z. The primary project under which this work was performed was funded with federal funds from the Division of Microbiology and Infectious Diseases, National Institute of Allergy and Infectious Diseases, National Institutes of Health, Department of Health and Human Services, under contract \#HHSN2722000900050C, "TB Clinical Diagnostics Research Consortium." The manuscript contents are solely the responsibility of the authors and do not necessarily represent the official views of the supporting offices.

\section{Availability of data and materials}

The datasets used and/or analyzed during the current study are available from the corresponding author on reasonable request.

\section{Ethics approval and consent to participate}

The scientific review board of the Infectious Diseases Institute (IDI), the Institutional Review Board (IRB) of the Joint Clinical Research Centre, Kampala, Uganda and the Uganda National Council for Science and Technology approved the study. All participants provided written informed consent before participation in the study. All consent forms were approved by the above mentioned ethics committees and IRBs.

\section{Consent for publication}

Not applicable.

\section{Competing interests}

The authors declare that they have no competing interests.

\section{Author details}

${ }^{1}$ Research Department, Infectious Diseases Institute, Makerere University College of Health Sciences, Kampala, Uganda. ${ }^{2}$ Department of Medicine, School of Medicine, Makerere University College of Health Sciences, Kampala, Uganda. ${ }^{3}$ Department of Medical Microbiology and Immunology, School of Biomedical Sciences, Makerere University College of Health Sciences, Kampala, Uganda. ${ }^{4}$ Johns Hopkins University School of Medicine, Baltimore, MD, USA.

\section{Received: 26 September 2020 Accepted: 27 December 2020} Published online: 13 January 2021

\section{References}

1. World Health Organization. Global tuberculosis report 2019. World Health Organization; 2019. Available at https://www.who.int/teams/globaltuberculosis-programme/tb-reports; Visited on June 30, 2020

2. Gelaw YA, Williams G, Soares Magalhaes RJ, Gilks CF, Assefa Y. HIV Prevalence Among Tuberculosis Patients in Sub-Saharan Africa: A Systematic Review and Meta-analysis. AIDS Behav. 2019;23(6):1561-75.

3. Lawn SD, Churchyard G. Epidemiology of HIV-associated tuberculosis. Curr OpinHIV AIDS. 2009;4(4):325-33

4. Gupta RK, Lucas SB, Fielding KL, Lawn SD. Prevalence of tuberculosis in post-mortem studies of HIV-infected adults and children in resource-limited settings: a systematic review and meta-analysis. AIDS. 2015;29(15):1987.

5. Uganda. Ministry of Health, Uganda National Tuberculosis and Leprosy Control Programme: Manual for management and control of tuberculosis and leprosy 3rd Edition, 2017, http://health.go.ug/sites/default/files/NTLP\%2 OManual\%203rd\%20edition_17th\%20Aug_final.pdf. Downloaded on June 26, 2020.

6. Henegar C, Behets F, Vanden Driessche K, Tabala M, Bahati E, Bola V, et al. Mortality among tuberculosis patients in the Democratic Republic of Congo. Int J Tuberc Lung Dis. 2012;16(9):1199-204.

7. Macpherson P, Dimairo M, Bandason T, Zezai A, Munyati SS, Butterworth $A E$, et al. Risk factors for mortality in smear-negative tuberculosis suspects: a cohort study in Harare, Zimbabwe. Int J Tuberc Lung Dis. 2011;15(10):1390-6.

8. Banda H, Kang'ombe C, Harries AD, Nyangulu DS, Whitty CJ, Wirima JJ, et al. Mortality rates and recurrent rates of tuberculosis in patients with smearnegative pulmonary tuberculosis and tuberculous pleural effusion who have completed treatment. Int J Tuberc Lung Dis. 2000:4(10):968-74.

9. Whitehorn J, Ayles H, Godfrey-Faussett P. Extra-pulmonary and smearnegative forms of tuberculosis are associated with treatment delay and hospitalisation. Int J Tuberc Lung Dis. 2010;14(6):741-4.
10. Steingart KR, Ng V, Henry M, Hopewell PC, Ramsay A, Cunningham J, et al Sputum processing methods to improve the sensitivity of smear microscopy for tuberculosis: a systematic review. Lancet Infect Dis. 2006; 6(10):664-74.

11. Lawn SD, Ayles H, Egwaga S, Williams B, Mukadi YD, Santos Filho ED, et al. Potential utility of empirical tuberculosis treatment for HIV-infected patients with advanced immunodeficiency in high TB-HIV burden settings. Int J Tuberc Lung Dis. 2011;15(3):287-95.

12. Manabe YC, Tenforde MW. Empirical tuberculosis therapy in advanced HIV disease. Lancet HIV. 2020;7(1):e3-5.

13. World Health Organization. The End TB Strategy. Global strategy and targets for tuberculosis prevention, care and control after 2015. In: WHO; 2015. Available at https://www.who.int/tb/strategy/end-tb/en/; Visited on June 30, 2020

14. Steingart KR, Sohn H, Schiller I, Kloda LA, Boehme CC, Pai M, et al. Xpert(R) MTB/RIF assay for pulmonary tuberculosis and rifampicin resistance in adults. Cochrane Database Syst Rev. 2013:1:CD009593.

15. Lawn SD, Mwaba P, Bates M, Piatek A, Alexander H, Marais BJ, et al. Advances in tuberculosis diagnostics: the Xpert MTB/RIF assay and future prospects for a point-of-care test. Lancet Infect Dis. 2013;13(4):349-61.

16. Parsons LM, Somoskövi Á, Gutierrez C, Lee E, Paramasivan C, Al A, et al. Laboratory diagnosis of tuberculosis in resource-poor countries: challenges and opportunities. Clin Microbiol Rev. 2011;24(2):314-50.

17. Kirwan DE, Cardenas MK, Gilman RH. Rapid implementation of new TB diagnostic tests: is it too soon for a global roll-out of Xpert MTB/RIF? Am J Trop Med Hyg. 2012;87(2):197-201

18. Vassall A, van Kampen S, Sohn H, Michael JS, John KR, den Boon S, et al. Rapid diagnosis of tuberculosis with the Xpert MTB/RIF assay in high burden countries: a cost-effectiveness analysis. PLoS Med. 2011;8(11): e1001120.

19. Steingart KR, Schiller I, Horne DJ, Pai M, Boehme CC, Dendukuri N. Xpert(R) MTB/RIF assay for pulmonary tuberculosis and rifampicin resistance in adults. Cochrane Database Syst Rev. 2014;1:CD009593.

20. World health Organization. Policy update: Xpert MTB/RIF assay for the diagnosis of pulmonary and extrapulmonary TB in adults and children. 2014.

21. World Health Organisation: Improving the diagnosis and treatment of smear-negative pulmonary and extra-pulmonary tuberculosis among adults and adolescents, Recommendations for HIV-prevalent and resourceconstrained settings, 13 March 2007, (WHO/HTM/HIV/2007.01). Available at http://www.who.int/hiv/pub/tb/pulmonary/en.

22. World Health Organization Chest radiography in tuberculosis detection summary of current WHO recommendations and guidance on programmatic approaches. 2016. Available at https:/apps.who.int/iris/ bitstream/handle/10665/252424/9789241511506-eng.pdf; Downloaded July 16,2020 .

23. Jaeger S, Karargyris A, Candemir S, Siegelman J, Folio L, Antani S, et al. Automatic screening for tuberculosis in chest radiographs: a survey. Quant Imaging Med Surg. 2013;3(2):89-99.

24. Davis JL, Worodria W, Kisembo H, Metcalfe JZ, Cattamanchi A, Kawooya M, et al. Clinical and radiographic factors do not accurately diagnose smearnegative tuberculosis in HIV-infected inpatients in Uganda: a cross-sectional study. PLoS One. 2010;5(3):e9859.

25. Greenberg SD, Frager D, Suster B, Walker S, Stavropoulos C, Rothpearl A. Active pulmonary tuberculosis in patients with AIDS: spectrum of radiographic findings (including a normal appearance). Radiology. 1994; 193(1):115-9.

26. Cudahy PGT, Dawson R, Allwood BW, Maartens G, Wilson D. Diagnostic outcomes after chest radiograph interpretation in patients with suspected tuberculosis and negative sputum smears in a high-burden human immunodeficiency virus and tuberculosis setting. Open forum infectious diseases. 2017;4(3):ofx123.

27. Nakiyingi L, Moodley VM, Manabe YC, Nicol MP, Holshouser M, Armstrong DT, et al. Diagnostic accuracy of a rapid urine lipoarabinomannan test for tuberculosis in HIV-infected adults. J Acquir Immune Defic Syndr. 2014;66(3): 270-9.

28. Nakiyingi L, Nonyane BA, Ssengooba W, Kirenga BJ, Nakanjako D, Lubega G, et al. Predictors for MTB Culture-Positivity among HIV-Infected SmearNegative Presumptive Tuberculosis Patients in Uganda: Application of New Tuberculosis Diagnostic Technology. PLoS One. 2015;10(7):e0133756.

29. Wilson D, Mbhele L, Badri M, Morroni C, Nachega J, Chaisson RE, et al. Evaluation of the World Health Organization algorithm for the diagnosis of 
HIV-associated sputum smear-negative tuberculosis. Int I Tuberc Lung Dis. 2011;15(7):919-24.

30. Kanaya AM, Glidden DV, Chambers HF. Identifying pulmonary tuberculosis in patients with negative sputum smear results. Chest. 2001;120(2):349-55.

31. Theron G, Pooran A, Peter J, van Zyl-Smit R, Kumar Mishra H, Meldau R, et al. Do adjunct tuberculosis tests, when combined with Xpert MTB/RIF, improve accuracy and the cost of diagnosis in a resource-poor setting? Eur Respir J. 2012;40(1):161-8.

\section{Publisher's Note}

Springer Nature remains neutral with regard to jurisdictional claims in published maps and institutional affiliations.

Ready to submit your research? Choose BMC and benefit from:

- fast, convenient online submission

- thorough peer review by experienced researchers in your field

- rapid publication on acceptance

- support for research data, including large and complex data types

- gold Open Access which fosters wider collaboration and increased citations

- maximum visibility for your research: over $100 \mathrm{M}$ website views per year

At $\mathrm{BMC}$, research is always in progress.

Learn more biomedcentral.com/submissions 